\title{
La construction des indicateurs de performance des services d'eau en France : mesurer le développement durable?
}

\author{
Guillem Canneva ${ }^{1}$, Lætitia Guérin-Schneider ${ }^{2}$ \\ 1 Sciences de gestion, AgroParisTech, UMR G-Eau, 34093 Montpellier cedex 5, France \\ 2 Sciences de gestion, CEMAGREF, UMR G-Eau, 34196 Montpellier cedex 5, France
}

\begin{abstract}
Ce texte aborde un enjeu scientifique et social important - la mesure du développement durable - et il le fait sur une problématique dont l'importance sociale n'est elle-même plus à démontrer : la gestion des services d'eau et d'assainissement. Fruit de l'analyse d'un cas approfondi sur lequel les auteurs ont fait une recherche-intervention, l'article aborde à la fois la substance des indicateurs élaborés et les caractéristiques du processus de négociation. Concernant les indicateurs, le lecteur de NSS retrouvera ici un phénomène déjà analysé dans ces colonnes : construire un indicateur, c'est faire le choix de rendre visibles certains phénomènes et donc d'en «invisibiliser » d'autres, en fonction d'une logique d'action et de compromis sociaux qu'il convient de décrypter. Concernant les procédures, l'inclusion des parties prenantes a surtout l'avantage de mettre la démarche en conformité avec l'un des canons du développement durable. Une illustration parmi d'autres de l'usage opportuniste auquel ce concept est parfois et trop souvent soumis..
\end{abstract}

La Rédaction

\section{Mots-clés :}

développement durable ; service public ; indicateurs ; convention ; régulation

\section{Keywords:}

sustainable development; public utilities; indicators; conventions; regulation

Résumé - Dans un contexte de défiance des usagers face à leurs services d'eau, la quantification de la performance par des indicateurs s'est construite par synthèse des contributions des parties prenantes. L'analyse de ce processus illustre les tractations autour de la définition des indicateurs. Malgré leur technicité et leur objectivité apparente, ils reflètent des intérêts particuliers et ne garantissent aucune neutralité. On appréhende ainsi comment les difficultés de la mesure et les emplois attendus des indicateurs influent sur leur définition. En effet, les enjeux contrastés entre les usages de contrôle et d'information ont été le principal moteur de la sélection du panel. Le développement durable apparaît, in fine, comme une légitimation a posteriori de la démarche, qui peut se prévaloir d'aborder des dimensions non financières et $\mathrm{d}^{\prime}$ inclure diverses parties prenantes.

\begin{abstract}
The construction of performance indicators for French water services: do they measure sustainable development? French water services have been characterized by a high level of fragmentation of local authorities and have often been delegated to private operators. During the 90 s price increases and corruption brought water utilities under the spotlight. Performance indicators (PI) then appeared to be an interesting tool for restoring trust and drawing attention to complementary operation aspects. As the creation of a national regulation authority was being debated, many stakeholder working groups developed their own PI selections. Despite the technical nature of the PI and its seeming objectivity, they reflect particular interests and do not actually guarantee any neutrality. The diversity of initiatives underlines the two main uses of external reporting: control or information. Even after the French regulation reform was abandoned (2002), the PI definition converged with a new law (2007). Our analysis allows us to understand how measurement difficulties and the expected uses of indicators influence their own definition. As a result, sustainable development can be seen as an ex post legitimization of the process rather than a real building framework, even though stakeholder participation and non-financial dimensions are taken into account.
\end{abstract}

\section{Introduction}

Les services d'eau et d'assainissement sont une composante essentielle du fait urbain. Ils constituent une

Auteur correspondant: G. Canneva, guillem.canneva@agroparistech.fr interface à la fois avec l'environnement, en y puisant la ressource et en y rejetant les eaux usées, et avec la société, en contribuant à son développement et à sa cohésion. En France, la réglementation a fixé depuis 2007 une liste de 27 indicateurs de performance que ces services doivent établir annuellement et présenter à leurs usagers. 
Le ministère de l'Écologie et du Développement durable, à l'initiative de la promulgation de cette liste, la présente in fine comme un outil d'évaluation du développement durable ${ }^{1}$.

La définition de la performance en vue de sa quantification ne va pas de soi. Le terme est polysémique et, si nous définissons la performance comme un résultat mesurable atteint par une organisation, se pose la question de la mesure mais également, au préalable, de ce qu'on mesure. Les services publics d'eau recouvrent des attentes plurielles et des intérêts économiques divergents. L'élaboration des indicateurs de performance représente donc un exemple intéressant d'une démarche de « conventionnement » (Desrosières, 2008), autour du concept de service d'eau bien géré. La logique de développement durable a-t-elle réellement été centrale dans ce conventionnement? Pour répondre, il faut analyser par qui ces indicateurs ont été définis et quels ont été les déterminants de leur élaboration. Historiquement, ils naissent dans un contexte de défiance des usagers face à la flambée des prix et, bien qu'un consensus se dégage finalement sur leur utilité, ils n'en sont pas moins l'objet d'âpres mais discrètes négociations entre parties prenantes.

Nous présenterons d'abord l'émergence du besoin de quantification, inscrite dans ce débat sur la régulation, avant d'analyser les étapes d'élaboration des indicateurs de performance. Enfin, en décryptant les définitions effectivement retenues, nous illustrerons leur caractère conventionnel selon les options stratégiques. Si le développement durable est affiché a posteriori comme structurant, la prise en compte réelle des trois dimensions, économique, sociale et environnementale, est tributaire du processus d'élaboration. En nous appuyant sur le positionnement original d'un des auteurs, acteur direct de la production des indicateurs ${ }^{2}$, nous analysons non seulement le contexte et la pression sociale expliquant l'émergence d'indicateurs, mais aussi l'influence et la finalité de différents types d'acteurs dans le processus. Cette analyse in situ permet de discuter le poids réel de la logique de développement durable face à d'autres ressorts plus

\footnotetext{
${ }^{1}$ Cf. annexe II de l'arrêté du 2 mai 2007 relatif aux rapports annuels sur le prix et la qualité des services publics d'eau potable et d'assainissement (http://www.legifrance.gouv.fr/affichTexte. do?cidTexte=JORFTEXT000000274838\&dateTexte $=$ ).

2 Cet article s'appuie sur une recherche-intervention réalisée au sein du laboratoire Gestion de l'eau et de l'assainissement d'AgroParisTech-Engref. La recherche-intervention (Moisdon, 1984 ; David, 2008) pose que théorie et pratique sont méthodologiquement liées dans un processus, en partie commun, de génération de connaissances. Les chercheurs ont été au cœur de l'élaboration des nouveaux indicateurs analysés : animation du travail sur les indicateurs au sein du réseau «Gestion des services publics » (GSP); participation aux groupes AFNOR, ISO, IGD, FNCCR (ces groupes sont détaillés dans le corps de l'article); appui technique à la mission d'inspection.
}

classiques du reporting ${ }^{3}$ : information externe et contrôle d'un monopole.

\section{L'émergence du besoin de quantification de la performance}

\section{Contexte institutionnel des services d'eau et d'assainissement en France}

Les enjeux de quantification de la performance s'ancrent dans un contexte institutionnel particulier. Les réseaux d'eau et d'assainissement collectif constituent des monopoles naturels associés à des externalités sur l'environnement et la santé. En France, les services d'eau potable et d'assainissement sont définis comme des services publics locaux à caractère industriel et commercial. Services publics, ils doivent respecter des principes essentiels de cohésion sociale (continuité et égalité de traitement), tout en s'adaptant à l'évolution des technologies et des attentes des usagers (mutabilité). Locaux, ils sont placés sous la responsabilité des communes. Même si celles-ci ont pu se regrouper en intercommunalités, le nombre de collectivités compétentes est demeuré important (Canneva et Pezon, 2008). Le caractère industriel et commercial oblige le service à équilibrer ses dépenses par les redevances payées par les usagers, sans recours à des ressources fiscales ${ }^{4}$. La collectivité compétente peut confier le service à un délégataire, qui se rémunère alors directement auprès des usagers. La gestion déléguée est prépondérante en France $(71 \%$ de la population desservie en eau potable et $56 \%$ en assainissement) et le secteur privé est fortement concentré : trois opérateurs principaux (Veolia Environnement, Suez Environnement et SAUR) détiennent plus de $97 \%$ du marché (Barucq et al., 2010).

À la suite de quelques affaires retentissantes de corruption, la délégation de service public a été encadrée : interdiction de la tacite reconduction (loi Sapin ${ }^{5}$ ) et limitation de la durée des contrats à 20 ans (loi Barnier ${ }^{6}$ ). La procédure de délégation est codifiée (loi Sapin), imposant la transparence et un formalisme propice à la concurrence, même si l'intuitu personae, c'est-à-dire le libre choix

\footnotetext{
${ }^{3}$ Le reporting est l'opération consistant, pour une organisation, à rendre compte de son activité, au travers de rapports et de bilans périodiques destinés à informer les personnes chargées de superviser l'activité, en interne ou en externe, et, plus largement, celles concernées par ses résultats.

${ }^{4}$ Les communes de moins de 3000 habitants et leurs regroupements bénéficient d'une exception à cette obligation (art. L. 2224-2 du Code général des collectivités territoriales).

${ }^{5}$ Loi n $93-122$ du 29 janvier 1993 relative à la prévention de la corruption et à la transparence de la vie économique et des procédures publiques.

${ }^{6}$ Loi n 95-101 du 2 février 1995 relative au renforcement de la protection de l'environnement.
} 
par les élus, n'est pas foncièrement remis en cause. La loi Barnier oblige également les collectivités compétentes à établir un rapport annuel sur le prix et la qualité du service (RPQS), destiné " notamment à l'information des usagers ». Le rôle des usagers dans la gouvernance des services au travers des commissions consultatives des services publics locaux (CCSPL) reste cependant limité.

Parallèlement, le débat institutionnel sur la régulation des services d'eau prend un essor important, comme en attestent de nombreux rapports (Cour des comptes, 1997; Haut Conseil du secteur public, 1999; Martinand, 2001; Tavernier, 2001 ; Cour des comptes, 2003). La plupart soulignent le manque de transparence et de concurrence et concluent sur le besoin de renouveler les outils de régulation, notamment via une autorité nationale. En 1998, D. Voynet, alors ministre de l'Environnement, propose de créer un Haut Conseil du service de l'eau et de l'assainissement (HCSEA) ; le projet est cependant abandonné lors du changement de majorité en mai 2002. Ce contexte influence néanmoins le développement des indicateurs de performance.

Le modèle de gestion des services d'eau en France est donc marqué par une asymétrie forte entre des opérateurs puissants et des collectivités morcelées, notamment en milieu rural, et par une absence de régulateur sectoriel et national fort au côté d'institutions non coordonnées (Conseil d'État, Conseil de la concurrence, Cour des comptes, Direction générale de la santé, etc.). Cependant, les collectivités ont toujours pu s'appuyer sur des sociétés d'ingénierie, des conseillers indépendants ou, jusque récemment, les services déconcentrés de l'État.

\section{L'approche historique de la performance du service d'eau et d'assainissement : le raccordement puis le prix}

Historiquement, la rémunération du service par les usagers a incité les opérateurs à les raccorder rapidement une fois les travaux réalisés. Après 1945, l'accès à l'eau s'est généralisé dans les zones rurales grâce aux subventions. Pendant plus de trente ans, la performance du service a reposé sur sa capacité à desservir le plus grand nombre.

À partir des années 1980, une conjonction de facteurs entraîne une augmentation significative de la facture d'eau. Associé à une dégradation de la ressource, le renforcement des normes sur la qualité de l'eau potable, puis sur l'assainissement collectif, impose de nouveaux investissements. Ensuite, le besoin de renouvellement des réseaux d'eau potable dans les villes, confrontées à un endettement important, conduit nombre d'entre elles à déléguer à des entreprises privées la gestion des services, en incluant d'importants investissements à la charge de ces opérateurs ou le paiement de droits d'entrée, qui sont alors répercutés sur les usagers. En outre, les contraintes d'équilibre budgétaire se renforcent parallèlement à la libération des prix, conduisant les collectivités à augmenter les recettes et donc les tarifs (Pezon et Canneva, 2009). Enfin, l'augmentation des redevances payées aux agences de l'eau vient encore alourdir la facture pour l'usager.

Dans ce contexte, le prix devient un indicateur de performance du service, garant d'une gestion efficace et image d'une absence de marge abusive au détriment des consommateurs. On voit alors proliférer les observatoires du prix de l'eau et les palmarès reviennent régulièrement dans la presse.

\section{D'une approche focalisée sur le prix aux indicateurs de performance}

Cependant, la comparaison sur le critère du seul prix induit des effets pervers. Le prix, fixé à l'échelle communale ou intercommunale, reflète les coûts liés aux conditions locales d'exploitation du service. Comparer les prix stigmatise injustement des services subissant les plus fortes contraintes. En outre, les services bénéficiant de subventions communales pour équilibrer leur budget $^{7}$ ou ne pratiquant pas correctement l'amortissement comptable peuvent afficher un tarif moins élevé. Surtout, en se focalisant sur le prix immédiat, on risque de négliger la gestion patrimoniale, indispensable à la pérennité du service. Les canalisations sont des infrastructures coûteuses et d'une durée de vie élevée, mais pas infinie. Une collectivité souhaitant réduire le prix de l'eau pourrait ne pas les renouveler régulièrement, les laisser se dégrader progressivement et reporter sur les générations futures les investissements nécessaires.

L'entrée unique par le prix est donc contestable. Elle peut être complétée par le contrôle des coûts. Toutefois, l'analyse des comptes du délégataire pour constater le niveau de la marge a aussi ses limites. Une partie non négligeable de l'activité de l'opérateur (gestion de la clientèle, services d'appui) est mutualisée entre plusieurs contrats. Afin de présenter des comptes de la délégation à chaque collectivité, les opérateurs répartissent les coûts mutualisés selon des clés internes. Ils font de plus intervenir des calculs extracomptables ne retranscrivant pas exactement les dépenses effectives de renouvellement. Ils rendent ainsi le contrôle à l'échelle de la collectivité complexe (Guérin-Schneider et Nakhla, 2000). En outre, par le jeu de sous-traitance entre filiales du même groupe (réparation de fuites, renouvellement de réseau), une partie de la marge peut être transférée et ainsi ne pas apparaître dans les comptes à l'échelle d'un contrat.

Face aux limites du contrôle des moyens, une approche fondée sur les résultats semble alors prometteuse

7 En application des exceptions prévues par l'art. L. 2224-2 du Code général des collectivités territoriales (cf. supra, note 4 ). 


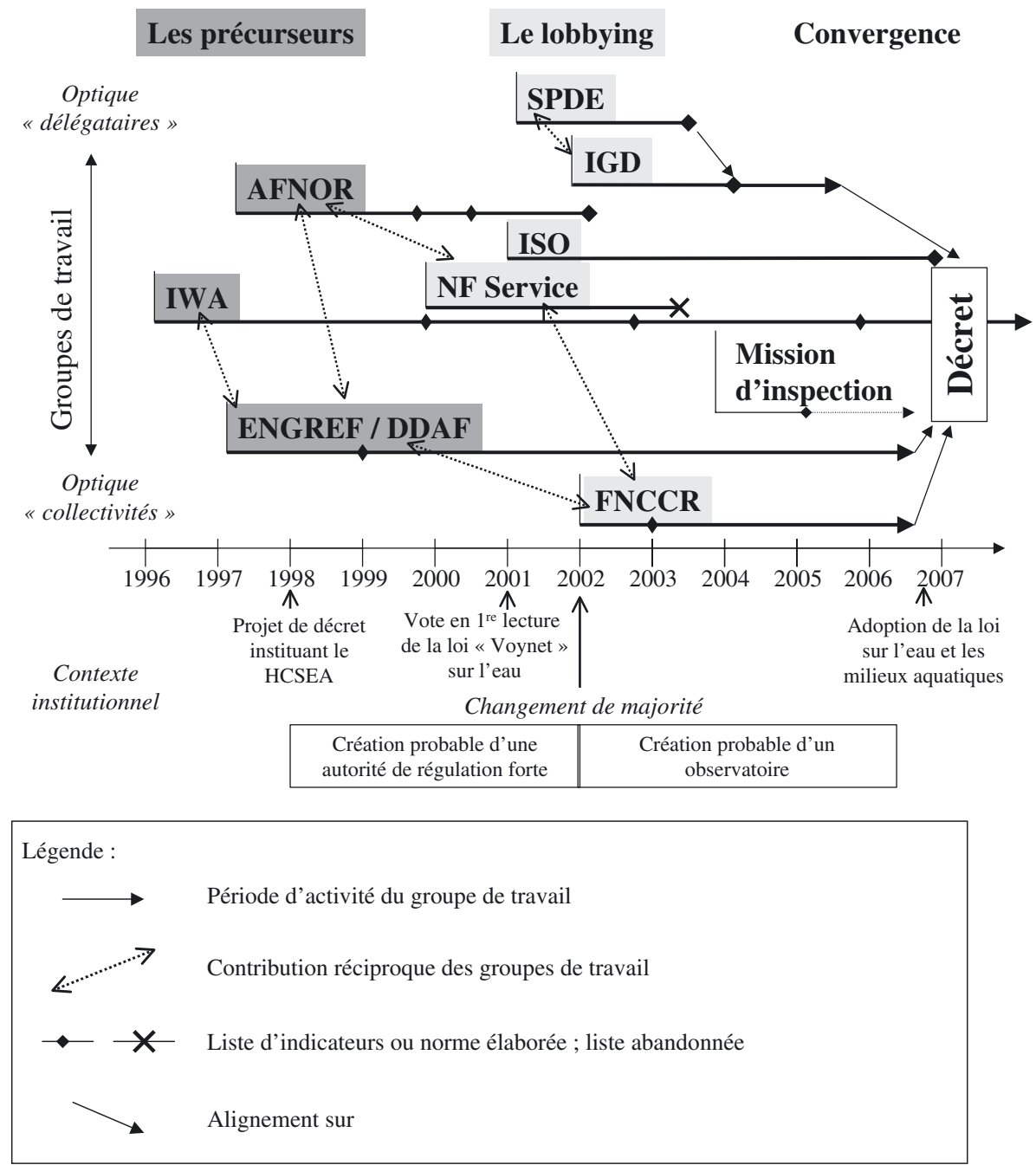

Fig. 1. Présentation chronologique des groupes de travail contribuant à l'élaboration des indicateurs de performance, en lien avec le contexte institutionnel.

pour restaurer la confiance entre usagers, élus et opérateurs. Il s'agit d'établir un contrat d'objectifs assignant des performances à atteindre. Charge au délégataire d'optimiser ses moyens pour en retirer des bénéfices. La régulation par les résultats passe par la définition des performances à atteindre et par leur mesure. Cela nécessite des indicateurs de performance établis en commun et reflétant la pluralité des fonctions de l'opérateur (satisfaction de la clientèle, entretien du réseau, exploitation efficace des équipements, etc.).

\section{Les étapes de la construction des indicateurs de performance}

Depuis la fin des années 1990, l'intérêt d'une régulation est partagé y compris par les entreprises, qui y voient un moyen de redorer leur blason. Commence alors un processus de construction qui débouche sur une réglementation imposant une liste commune d'in- dicateurs à intégrer dans les RPQS. Ce processus n'est cependant pas linéaire. Les indicateurs de performance ont été construits par des groupes de travail aux motivations contrastées, voire contradictoires. Trois temps se dégagent : celui des précurseurs ; celui du lobbying ; puis celui de la convergence (Fig. 1). La participation d'un des auteurs aux différents groupes (Guérin-Schneider, 2001; Guérin-Schneider et Nakhla, 2003) permet d'analyser les ressorts en jeu dans les travaux préparatoires : quels ont été les principaux contributeurs? quelles étaient les finalités de leurs travaux? Cette décomposition des stratégies, où la dimension du développement durable n'est que tardivement explicitée, interroge l'affichage final du décret fixant les indicateurs de performance.

\section{Les précurseurs}

Ces groupes de travail se sont constitués en amont du processus, avec le plus souvent une légitimité technique ou scientifique forte. Ils structurent la définition 
de la performance et construisent les premiers indicateurs. Leur positionnement stratégique est néanmoins assez différencié.

Le plus ancien (Alegre et Baptista, 1997) est le groupe de l'Association internationale de l'eau (en anglais, IWA). Il vise à définir des indicateurs de performance communs, techniques et financiers, comme outil de management. La comparaison des performances entre organisations permet d'améliorer la gestion selon le principe du benchmarking (Camp, 1989). L'usage de tels indicateurs comme outil de gouvernance externe pour la régulation est mentionné comme une application possible, mais la finalité première est interne et opérationnelle. Ces travaux débouchent sur des manuels techniques détaillés et complets, comportant plusieurs centaines d'indicateurs (Alegre et al., 2000 ; Matos et al., 2003).

$\mathrm{Au}$ niveau français, l'AFNOR cherche à créer des normes de services relatives aux services d'eau et d'assainissement. Cette initiative, fortement soutenue par les opérateurs privés, mobilise également les collectivités (notamment la Fédération nationale des collectivités concédantes et régies [FNCCR]) et des associations d'usagers. Il s'agit de définir les propriétés d'un «bon » service, c'est-à-dire capable de satisfaire les attentes des usagers. Les normes déclinent des missions et proposent des indicateurs, qui restent à un niveau de précision succinct. Elles définissent également des outils méthodologiques pour mesurer la performance. Ainsi les normes de l'AFNOR précisent la distinction entre les informations de contexte (" caractéristiques de réseau et paramètres de complexité »), les indicateurs relatifs aux moyens évaluant une opération d'exploitation («indicateurs d'activité ») et les indicateurs évaluant un critère de qualité («indicateur de performance »). Lancées en 1997, les normes sont promulguées de 2000 à 2003, sans pour autant atteindre leur finalité ultime : créer une marque de qualité NF services et un référentiel de certification. Si l'objectif affiché de ce processus est de mieux répondre aux attentes des clients à un coût maîtrisé (Olivier et Moutet, 2002), les entreprises dominantes cherchent également à asseoir leur position en faisant reconnaître leur mode d'organisation comme un standard (Giard, 2003). Elles tentent ainsi de revaloriser leur image écornée par les scandales et de contrer l'émergence de concurrents.

Enfin, des travaux de recherche en gestion publique à l'École nationale du génie rural, des eaux et des forêts (ENGREF) [Guérin-Schneider, 2001], qui débutent à la même période, se focalisent sur l'utilisation des indicateurs comme instruments de régulation locale par les collectivités et sont menés en lien avec les praticiens. Cette recherche a émergé notamment de l'expérience des ingénieurs des directions départementales de l'Agriculture et de la Forêt (DDAF) qui interviennent comme conseillers des collectivités face aux délégataires et sont regroupés dans le réseau thématique «Gestion des services publics »
(GSP). Outre les résultats académiques (thèse), ces travaux débouchent sur l'introduction d'indicateurs de performance dans le modèle de contrat de délégation des DDAF et le développement d'un logiciel pour le suivi de la performance. Ces travaux vont susciter l'intérêt du ministère de l'Écologie et du Développement durable, qui envisage aussi depuis plusieurs années de renforcer la régulation du secteur.

\section{Le foisonnement du lobbying}

Dès que D. Voynet officialise son projet de régulateur national, en 1998, il apparaît clairement que les indicateurs vont devenir un instrument du futur régulateur, en réponse au besoin de transparence et d'incitation. Les principales parties prenantes vont reprendre à leur compte le développement d'indicateurs afin d'avoir une chance d'influencer le référentiel officiel qui s'annonce. Les innovations des précurseurs sont diffusées, testées sur le terrain et confrontées aux différents points de vue. Après quelques années, des groupes de travail pilotés par différents lobbies sont formellement mis en place.

Un premier groupe, piloté par l'Institut de la gestion déléguée (IGD), est formé fin 2001 par les entreprises privées. Il associe des experts (dont l'ENGREF), des représentants des collectivités (dont la FNCCR) et des représentants des usagers. Sa légitimité est plus forte que celle d'un second groupe, constitué uniquement d'entreprises et piloté par le Syndicat professionnel des distributeurs d'eau (SPDE). Les entreprises, après une tentative unilatérale pour imposer la liste SPDE, finiront d'ailleurs par s'aligner sur celle de l'IGD, plus consensuelle et plus riche.

Début 2002, un autre groupe se constitue autour de la FNCCR, associant des collectivités en délégation et en régie pour représenter clairement l'intérêt des collectivités. Il s'appuie sur les travaux de l'ENGREF et de l'AFNOR pour développer sa propre liste d'indicateurs.

Dans la suite des normes de service, un nouveau groupe de travail de l'AFNOR échoue à créer un référentiel pour une marque NF. Cependant, le principe des normes est repris en 2001, sur proposition française, au sein de l'International Standard Organisation (ISO). Perçue par de nombreux pays comme une tentative de standardiser la gestion de l'eau suivant le modèle français, cette initiative génère des négociations importantes qui éloignent les nouvelles normes internationales de l'esprit initial de la norme de service, pour tendre vers des normes de processus, établissant une méthode d'amélioration continue.

Jusqu'en 2002, le projet Voynet, avec la perspective d'un régulateur fort, reste d'actualité. Le ministère chargé de l'Environnement contribue au processus de réflexion, notamment en finançant les travaux de la FNCCR et de l'ISO. 

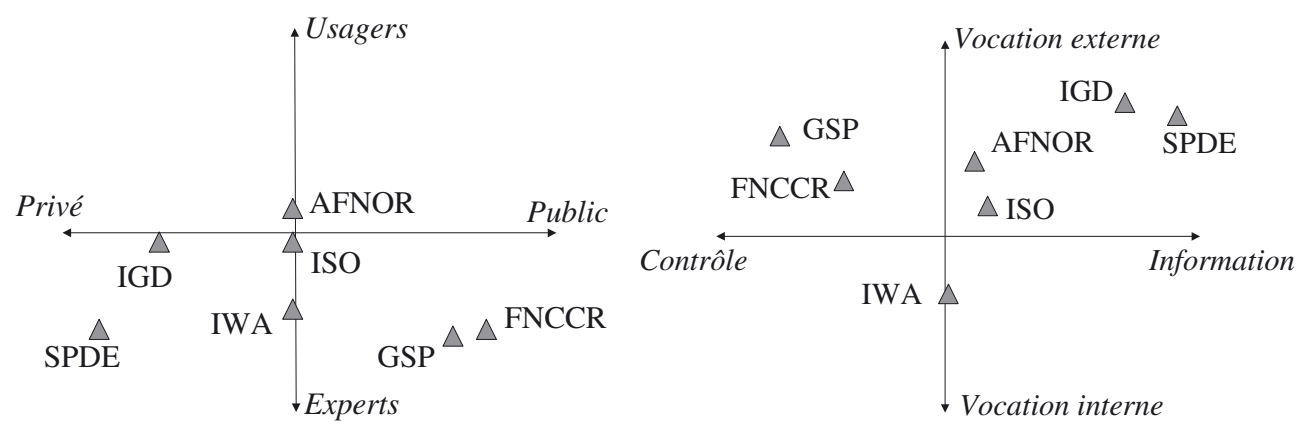

Fig. 2. Positionnement des différents groupes de travail suivant la nature des contributeurs et la finalité des travaux.

Notre participation à plusieurs groupes de travail entre 1997 et 2006 nous permet de les positionner en fonction de leur composition et de leurs perspectives d'utilisation des indicateurs de performance (Fig. 2). Ainsi, les contributeurs principaux relèvent de la sphère publique (collectivités, services de l'État et laboratoires de recherche publics) ou privée (entreprises, délégataires de service) et se positionnent en tant qu'experts (ingénieurs, chercheurs ou spécialistes du reporting) ou, à l'opposé, s'ouvrent aux usagers. Parallèlement, les groupes visent un objectif de régulation ou de restauration d'image, dans une perspective interne de recherche d'efficience ou, externe, de reporting. Ces axes stratégiques ambivalents ont déjà été mis en évidence par de nombreux travaux sur l'utilisation des normes techniques ou du reporting volontaire non financier (Sayre, 1996 ; Quairel, 2004 ; Lakhal, 2006; Janicot, 2007).

Un lien fort apparaît entre le positionnement des contributeurs et l'objectif poursuivi, ce qui identifie des groupes stratégiquement proches. D'un coté, les contributeurs publics (GSP, FNCCR) visent essentiellement le contrôle externe. La communication vers les usagers et l'amélioration de la gestion interne via la comparaison des pratiques, sans être absente du groupe FNCCR, restent secondaires. À l'opposé, l'IGD et le SPDE, liés aux entreprises privées, donnent un poids plus fort à la communication externe. Néanmoins, l'IGD, qui se veut plus consensuel, n'exclut pas une utilisation des indicateurs de performance pour le suivi par les collectivités. Entre les deux, les travaux de normalisation (AFNOR et ISO) impliquent une diversité plus grande de parties prenantes et intègrent des objectifs ambivalents, entre gages de bonnes pratiques et outils de communication, même si ces derniers prédominent. L'IWA est le seul groupe pour lequel l'utilisation des indicateurs est essentiellement interne : il s'agit d'améliorer les propres processus de l'organisation, par comparaison avec d'autres (process benchmarking).

On constate également une dominance des experts sur les abonnés dans les groupes de travail. Les associations de consommateurs ne sont représentées significativement que dans les groupes de normalisation (ISO et AFNOR) et à l'IGD.
Lors des travaux, la notion de développement durable a été peu, voire pas du tout évoquée, même si la logique consiste clairement à élaborer des indicateurs prenant en compte les dimensions non financières. Les travaux s'appuient plutôt sur une décomposition des fonctions à assumer par le service (missions de service public et gestion opérationnelle et patrimoniale), comme en attestent les classifications proposées pour les indicateurs dans les différents groupes.

\section{La convergence}

La multiplicité des groupes de travail masque une convergence sur un grand nombre de définitions. Outre l'influence commune des précurseurs, chaque groupe a cherché à associer les autres pour obtenir un consensus et une légitimité (Fig. 1). Certains acteurs vont jouer le rôle de passeur entre les groupes, en particulier L. GuérinSchneider, chercheur à l'ENGREF et membre du réseau GSP, qui participe à l'ensemble des groupes, excepté celui du SPDE. Le chef du service de l'eau à la FNCCR est également impliqué dans tous les groupes sauf l'IWA et le SPDE. Cependant, tant que ces indicateurs gardaient un statut de démarche volontaire issue d'initiatives particulières, le risque était grand de voir à terme les définitions diverger.

Alors que la majorité politique change en 2002 et que le projet de régulateur national est abandonné, le ministère de l'Écologie et du Développement durable commande néanmoins une mission d'inspection chargée de synthétiser les démarches en cours et de sélectionner les indicateurs de performance jugés les plus utiles et les plus fiables (Cousquer et al., 2005). Les fonctionnaires de la direction de $l^{\prime} \mathrm{Eau}^{8}$ qui avaient travaillé à la rédaction du premier projet de loi sur l'eau de D. Voynet restent en place et vont contribuer à maintenir les indicateurs de performance à l'agenda politique. À nouveau, les passeurs vont favoriser l'intégration des démarches : L. Guérin-Schneider est sollicitée par son ministère de tutelle, l'Agriculture, pour un appui technique à la mission

\footnotetext{
${ }^{8}$ Notamment le bureau de la directive-cadre et de la programmation, qui a appuyé les travaux de L. Guérin-Schneider (ceux effectués dans le cadre du réseau GSP), de la FNCCR et de l'AFNOR.
} 
Cousquer. On y retrouve un ingénieur en chef membre du réseau GSP (J. Dumont). La mission rencontre l'ensemble des groupes ainsi que des associations de consommateurs et propose finalement une liste d'indicateurs, pour la plupart communs à plusieurs groupes.

On aboutit ainsi à une liste qui concilie différentes finalités. Le rapport indique explicitement obéir à deux lignes directrices : «La première exprime une démarche locale de mesure de la performance, impliquant le développement de la relation à l'usager. La seconde correspond au souci d'initier des démarches de progrès » (Cousquer et al., 2005, p. 8). Mais la prégnance des contributions techniques reste forte, ce qui limite la pertinence de la liste pour la communication aux usagers. La perspective de la régulation est toujours présente dans le rapport, qui envisage explicitement la mise en place d'un observatoire national. S'il est cité en introduction du rapport Cousquer, le développement durable n'intervient cependant pas directement dans la structuration de la liste, qui reprend l'architecture des travaux précédents (qualité de service, pérennité du patrimoine, gestion économique et financière).

En fin de compte, la liste intègre à la fois des préoccupations traditionnelles (continuité du service ou qualité du traitement) et d'autres, plus modernes, qui renvoient aux différents visages de l'usager (Pflieger, 2002) ; des indicateurs sur le prix du service pour l'usagerconsommateur; des indicateurs sur la qualité des prestations immédiates (respect des délais), qui renvoient à l'usager-client; des indicateurs liés à l'environnement et à la gestion du patrimoine, qui interpellent l'usagercitoyen.

Le ministère s'appuie sur ce rapport pour préparer le décret et l'arrêté du 2 mai 2007, portant sur les indicateurs de performance à produire dans le RPQS (cf. Tab.). Les discussions finales sont conduites avec la FNCCR et le $\mathrm{SPDE}^{9}$. L'affichage politique a largement évolué : 1'enjeu d'information de l'usager l'emporte formellement sur celui du contrôle. C'est aussi à ce moment-là que la notion de développement durable est explicitement citée comme un aspect central.

\section{La définition des indicateurs, entre délimita- tion et valorisation de la performance}

La plupart des démarches justifiaient leurs indicateurs en s'appuyant sur les grandes fonctions du service : prestation immédiate aux usagers, continuité du service, pérennité du patrimoine et préservation de l'environnement et de la santé publique. En outre, les indicateurs ont été choisis pour leurs qualités opérationnelles : être précis, représentatifs et faciles à interpréter, couvrir la

\footnotetext{
${ }^{9}$ Qui entre-temps est devenu la Fédération professionnelle des entreprises de l'eau (FP2E).
}

totalité des fonctions du service sans être redondants, être applicables sur tout le territoire et sans coût de mesure important. Ces considérations paraissent neutres car techniques et objectives. Pourtant, au cours du débat, les positions particulières s'expriment sur le poids à donner à la régulation ou sur la prise en compte des usagers. Sans être exhaustifs, nous présentons plusieurs choix de définitions qui laissent transparaître ces différentes optiques et traduisent le niveau d'influence des groupes de travail.

\section{L'attrait du « bon résultat chiffré »}

Le réseau constitue la pièce majeure du service d'eau, à la fois par sa fonction et par sa valeur financière. Un niveau de fuites limité traduit un réseau en bon état et performant, et donc un patrimoine qui garde sa valeur. Le décret comporte trois indicateurs de performance apparemment très proches, allant à l'encontre du principe de non-redondance : l'indice linéaire de pertes, l'indice des volumes non comptés et le rendement du réseau. On peut exprimer les pertes ( $p=$ entrées réseau-sorties réseau):

- soit de manière relative, sous la forme d'un rendement $(\%)$

$\rho=\frac{\text { sorties réseau }}{\text { entrées réseau }}=\frac{\text { entrées réseau-pertes }}{\text { entrées réseau }}=1-\frac{\text { pertes }}{\text { entrées réseau }}$

- soit de manière absolue, sous la forme d'un indice linéaire de pertes $\left(\mathrm{m}^{3} / \mathrm{km} /\right.$ jour $)$

$$
I=\frac{\text { pertes }}{\text { longueur du rseau }}
$$

Le rendement traduit la consommation de la ressource ; c'est avant tout un indicateur économique (productivité) ou environnemental (gaspillage de la ressource). Mais il n'est pas parfaitement adapté pour évaluer l'état du réseau ; en effet, plus la consommation augmente, plus le rendement s'améliore, alors que le niveau de pertes reste constant ${ }^{10}$. Le décret reprend logiquement le rendement comme indicateur environnemental.

Toutefois, la définition retenue intègre les ventes en gros, contrairement à ce qui avait été recommandé à l'origine (Cousquer et al., 2005). Si elles peuvent se faire théoriquement $\mathrm{n}$ 'importe où sur le réseau, elles ont lieu le plus souvent avant la distribution. Dans ce cas, la définition originelle est la plus juste; elle est traditionnellement utilisée dans les comptes rendus techniques des exploitants. La seconde définition, intégrant les ventes en gros dans les sorties de réseau, produit un résultat chiffré plus favorable (Fig. 3). En la retenant, le décret contribue

\footnotetext{
${ }^{10}$ Le niveau des pertes dans un réseau varie avec la pression et non avec la consommation. Pour une justification plus détaillée, se reporter à Guérin-Schneider (2001).
} 
Tableau. Liste des indicateurs de performance du décret du 2 mai 2007.

NB : Les indicateurs en italique sont ceux exigibles uniquement pour les services disposant d'une CCSPL, c'est-à-dire les services les plus importants. Les indicateurs en gras sont ceux mentionnés dans l'article.

\begin{tabular}{|c|c|}
\hline \multicolumn{2}{|c|}{$\begin{array}{l}\text { Taux moyen de renouvellement des réseaux (en assainissement, uniquement pour les services avec CCSPL) } \\
\text { Durée d'extinction de la dette de la collectivité } \\
\text { Taux d'impayés } \\
\text { Taux de réclamations } \\
\text { Indice de connaissance et de gestion patrimoniale de réseaux } \\
\text { Montant des abandons de créance ou versement à un fonds de solidarité } \\
\text { Taux de respect du délai maximal de branchement }\end{array}$} \\
\hline $\begin{array}{l}\quad \text { INDICATEURS SPÉCIFIQUES EAU POTABLE } \\
\text { Taux de conformité de l'eau distribuée (microbiologie et } \\
\text { paramètres physico-chimiques) } \\
\text { Rendement du réseau de distribution } \\
\text { Indice linéaire des volumes non comptés } \\
\text { Indice linéaire de pertes en réseau } \\
\text { Indice d'avancement de la protection de la ressource en } \\
\text { eau } \\
\text { Taux d'occurrence des interruptions de service non programmées }\end{array}$ & $\begin{array}{l}\text { INDICATEURS SPÉCIFIQUES ASSAINISSEMENT COLLECTIF } \\
\text { Taux de desserte } \\
\text { Conformité réglementaire de la collecte, des équipements } \\
\text { des ouvrages d'épuration et des performances des équipements } \\
\text { d'épuration } \\
\text { Taux de boues évacuées selon des filières conformes à la } \\
\text { réglementation } \\
\text { Taux de débordement des effluents dans les locaux des usagers } \\
\text { Nombre de points du réseau de collecte nécessitant des interven- } \\
\text { tions fréquentes de curage par } 100 \mathrm{~km} \text { de réseau } \\
\text { Indice de connaissance des rejets au milieu naturel par les } \\
\text { réseaux de collecte }\end{array}$ \\
\hline \multicolumn{2}{|l|}{ Liste des indicateurs descriptifs } \\
\hline \multicolumn{2}{|c|}{$\begin{array}{l}\text { Prix TTC du service au } \mathbf{m}^{3} \text { pour } 120 \mathrm{~m}^{3} \\
\text { Délai maximal d'ouverture du branchement pour les nouveaux abonnés }\end{array}$} \\
\hline
\end{tabular}

implicitement à donner une meilleure image de la performance, mais nuit, au moins transitoirement, au suivi de l'évolution du rendement et donc à la régulation.

Comme indicateur lié au patrimoine, le décret retient deux indices linéaires de pertes. Il existe là encore deux manières de mesurer : une définition juste du point de vue technique, mais imprécise en termes de mesure-l'indice linéaire de pertes (ILP) ; une définition qui s'appuie sur des mesures précises, mais surestime les fuites - l'indice linéaire des volumes non comptés (ILVNC).

L'ILVNC est obtenu en comparant les volumes mesurés à l'entrée du réseau et les volumes mesurés à la sortie par les compteurs des abonnés (ou de vente en gros). Il inclut donc dans les pertes tous les volumes consommés non comptés, y compris les usages autorisés (eaux de service, par exemple, pour le lavage des filtres en production, eau des fontaines publiques sans comptage, eau pour éteindre les incendies... ). Pour passer à l'ILP, ces volumes sont retranchés, ce qui suppose de les estimer.

À nouveau, la logique de contrôle et celle de communication se trouvent en contradiction. Pour un même service, l'ILVNC donne un moins bon résultat que l'ILP. Par contre, la mesure étant plus précise, l'évolution inter- annuelle est plus fiable pour traduire l'évolution de l'état du réseau, et donc la pérennité du patrimoine. L'ILVNC est par conséquent un indicateur approprié pour le contrôle externe, alors que l'ILP est plus satisfaisant pour donner une bonne image du service. C'est aussi l'indicateur utilisé dans les comparaisons internationales. Finalement, le ministère de l'Écologie et du Développement durable, sensible autant aux enjeux de régulation qu'aux comparaisons internationales, a conservé les deux.

Ces trois indicateurs illustrent donc clairement les tensions entre la logique d'information externe pour améliorer l'image de l'opérateur et celle de contrôle externe pour réguler le service.

\section{Le prix exclu du champ de la performance}

Certaines dimensions de la performance ont été sousexplorées : c'est le cas des éléments financiers. Les indicateurs de performance ne suivent ni les coûts ni les prix. Afin d'améliorer le contrôle, l'ENGREF avait tenté, sans succès, d'inclure des données sur les dépenses de renouvellement, qui ont des conséquences importantes sur le 


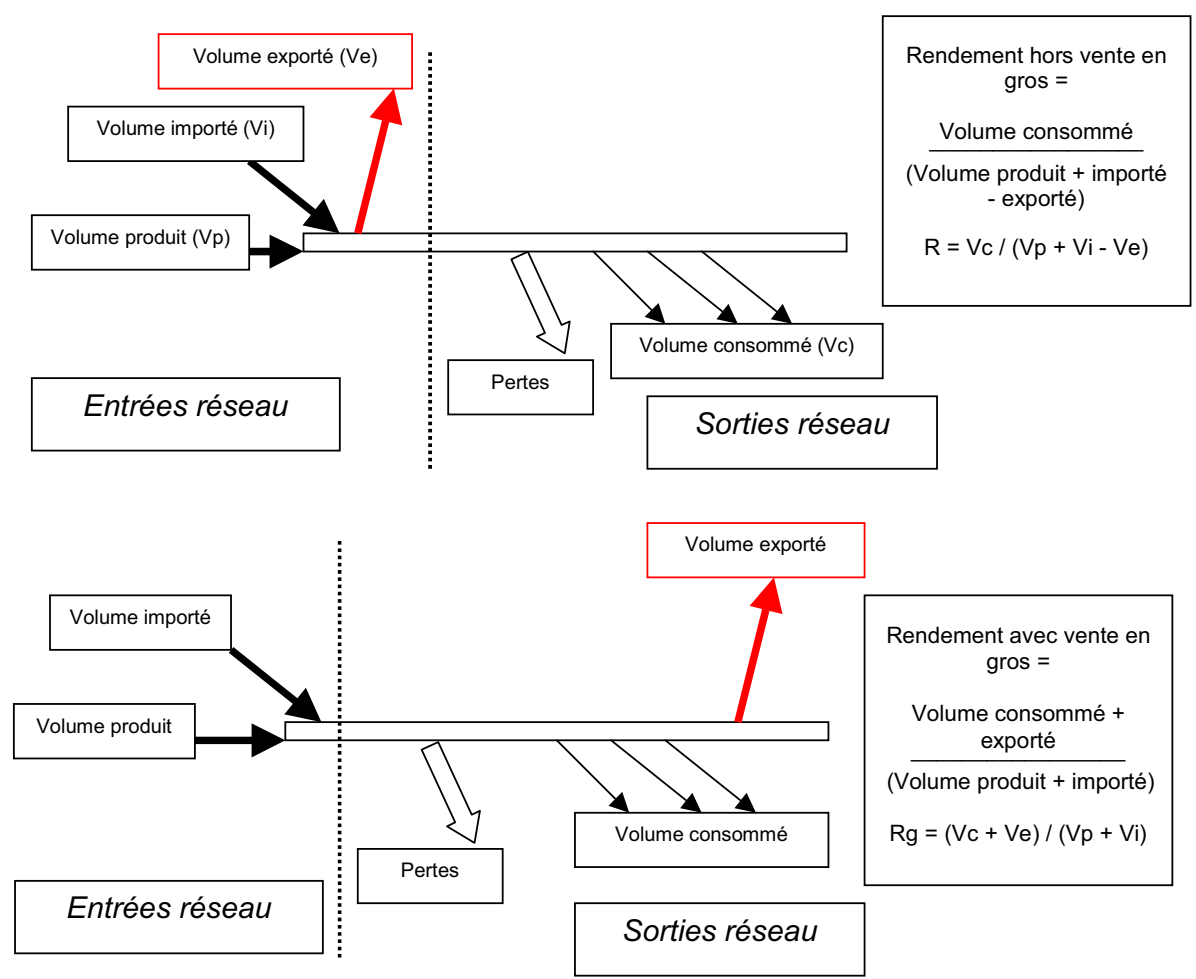

Fig. 3. Deux définitions usuelles du rendement, d'après Guérin-Schneider (2001).

prix et la pérennité du service. Les collectivités disposaient de peu d'instruments pour maîtriser cet aspect en cas de délégation. Un tel indicateur a toujours été refusé par les délégataires ${ }^{11}$. La FNCCR a introduit quelques indicateurs financiers (niveau d'épargne et endettement des budgets publics, taux d'impayés), sans pour autant inclure le prix dans les indicateurs. Le fait d'inclure le prix dans les caractéristiques descriptives pourrait sembler un arbitrage technique (cf. supra, les catégories définies dans les normes AFNOR), sans grande conséquence, dans la mesure où l'information descriptive est collectée au même titre que les indicateurs de performance. Pourtant, cela traduit la volonté d'afficher le prix comme étant un élément imposé par le contexte, sur lequel les gestionnaires (opérateurs et collectivités) n'auraient pas de prise, et ne pouvant se prêter à la comparaison entre services. Les délégataires ont été dans le même sens : ils ont argumenté que, la délégation étant un contrat d'objectif associé à une liberté de moyens, le prix devait être considéré comme une caractéristique descriptive du service et non comme une performance. Cette position a été finalement retenue dans le décret : le prix est une «information ».

D'autres cultures nationales sont beaucoup moins rétives à communiquer sur les coûts. Ainsi, en Angleterre,

\footnotetext{
11 Cependant, la loi n 2006-1772 du 30 décembre 2006 sur l'eau et les milieux aquatiques a repris l'idée d'un contrôle approfondi du renouvellement, obligeant à chiffrer en début de contrat un programme prévisionnel et à reverser en fin de contrat les sommes non dépensées à la collectivité.
}

le régulateur des services d'eau (Office of Water Services) a une vocation explicitement économique. Chargé de fixer le prix des services, il collecte et publie régulièrement une information détaillée sur les coûts en parallèle à des indicateurs sur le niveau de service. Le manuel de l'IWA comporte également un chapitre entier consacré aux indicateurs de coûts et aux recettes. En France, au contraire, les coûts ont toujours été considérés comme difficiles à mesurer (Burtin et al., à paraître). En outre, les perspectives de comparaison que fait naître le projet d'observatoire (finalement concrétisé dans la loi sur l'eau de 2006) incitent les opérateurs et les élus à ne pas s'exposer sur un thème sensible en termes d'image.

Le volet financier, à l'origine de la crise de confiance du secteur de l'eau, est ainsi resté tabou. Cela montre objectivement l'écart avec une application complète du développement durable qui s'y intéresse à la fois sous l'angle du recouvrement des coûts (dimension économique) et de l'acceptabilité du prix (dimension sociale).

\section{Les dimensions peu valorisantes écartées}

Une autre dimension, cette fois-ci technique, initialement présente dans plusieurs groupes de travail a disparu dans le décret : l'état du réseau d'assainissement et la production de boues d'épuration. Ces éléments sont pourtant fondamentaux, car ils traduisent l'efficacité de la dépollution du système d'assainissement. 
$\mathrm{Au}$ lieu de mesurer le taux de rejet dans le milieu, à travers un suivi des débordements et des fuites sur les réseaux, le décret propose simplement un «indice de connaissance des rejets au milieu naturel par les réseaux de collecte des eaux usées ». Il s'agit de savoir si le service dispose ou non des moyens de mesure des rejets. Le taux de production de boues, qui compare la quantité de boues produite (les matières polluantes extraites) à la quantité de boues théorique (celle qui aurait dû être extraite si la station fonctionnait bien), a été supprimé. Le taux d'eaux parasites ${ }^{12}$, qui permet de savoir si un réseau d'assainissement est en bon état, a également disparu alors qu'il était initialement présent. La mission d'inspection s'est justifiée en argumentant que les indicateurs élaborés n'étaient pas de précision et de fiabilité suffisantes. Ainsi, la «mesurabilité » de la performance a joué comme un facteur limitant l'exhaustivité de l'analyse. Cette situation est regrettable dans l'optique de la régulation, car les domaines laissés dans l'ombre sont probablement ceux où une marge de progrès importante existe. En effet, précédemment, les efforts ont surtout porté sur l'amélioration des stations d'épuration, laissant parfois de côté le renouvellement et la réhabilitation des réseaux $\mathrm{d}$ 'assainissement. Ces derniers se dégradent et peuvent générer des pollutions ou des dysfonctionnements. Il est donc peu valorisant de braquer un projecteur sur cet aspect. Pour le développement durable, c'est une dimension environnementale importante qui a été laissée de côté.

Enfin, les différents travaux sur les indicateurs de performance ont peu exploré la dimension sociale du service, notamment sa capacité à pourvoir aux besoins essentiels de tous, indépendamment de la capacité à payer. La construction du panel d'indicateurs confronte donc plusieurs périmètres de la performance : celle de l'opérateur, celle de la collectivité responsable du service ou celle de l'usager qui paie une facture, et exclut certaines dimensions pourtant essentielles dans le concept de développement durable.

\section{Conclusion}

En réaction à la crise de confiance des années 1990, le secteur des services d'eau a dépassé une approche de la performance focalisée sur le prix pour y intégrer des dimensions techniques, économiques et sociales, liées aux caractéristiques du service public. Après les travaux exploratoires, les différentes parties prenantes ont essayé d'infléchir l'élaboration d'indicateurs, puis la réglementation a figé les définitions à un niveau national.

Le développement durable apparaît comme une justification a posteriori de la démarche d'élaboration. En s'in-

\footnotetext{
${ }^{12}$ Ce sont les eaux qui n'auraient pas dû être collectées par le réseau d'assainissement (eau drainée, eaux pluviales, etc.).
}

téressant à des dimensions non financières et en convoquant les parties prenantes, celle-ci en arborait deux attributs essentiels (Faucheux et Nicolaï, 2004). Le choix des indicateurs de performance montre cependant que, sur la forme, la logique technique-expert a dominé et que, sur le fond, l'enjeu a surtout porté sur l'arbitrage entre régulation d'un monopole local et amélioration de l'image des opérateurs. Le développement durable serait devenu entre-temps un paradigme incontournable, mobilisé autant par les entreprises que par les organisations publiques pour répondre à un «engouement » (Rousseau, 2008). On peut également faire l'hypothèse que le développement durable légitime, in fine, la continuité d'action de la direction de l'Eau du ministère de l'Écologie et du Développement durable sur les indicateurs de performance, lui évitant d'être sapée par l'abandon d'une politique de régulation plus forte du secteur.

Cette trajectoire montre comment des indicateurs de performance, sous des atours techniques et impartiaux, sont en fait le reflet d'intérêts particuliers négociés. Leur évolution illustre comment les usages qu'ils laissent entrevoir - et notamment le spectre de la comparaison contraignent leur définition autour des éléments les plus consensuels, dont la mesure est la plus simple et dont les résultats valorisent la technicité de l'opérateur.

Selon Desrosières (2008, p.10), "quantifier, c'est convenir puis mesurer ». Si la formule lapidaire souligne la part de construction sociale dans une entreprise de réduction en nombre d'un concept, elle peut laisser croire à un processus séquentiel. Or, l'exemple développé montre que les parties prenantes anticipent les usages des indicateurs et essaient d'influencer leur choix et leurs définitions de sorte que leurs intérêts soient préservés. Cet exemple complète des résultats observés dans d'autres secteurs (Janicot, 2007). De telles démarches volontaires répondent souvent à deux enjeux contradictoires : celui du contrôle, porté par les parties prenantes externes; celui de la communication, porté par les parties prenantes internes.

La recherche-intervention s'est avérée une approche particulièrement féconde pour appréhender la dynamique de l'innovation managériale. Pour reprendre les notions développées par David (1996 et 2008), la participation active aux groupes de travail a permis à la fois de comprendre les mécanismes de formalisation (degré de précision dans la définition initiale de l'innovation) et de contextualisation (processus particulier de transformation réciproque de l'innovation par les acteurs et des acteurs par l'innovation).

\section{Références}

Alegre, H., Baptista, J.M. (Eds), 1997. Workshop Performance Indicators for Transmission and Distribution Systems, Lisbon, International Water Association. 
Alegre, H., Hirner, W., Baptista, J.M., Parena, R., 2000. Performance Indicators in Water Supply Systems: IWA Manual of Best Practice, London, IWA Publishing.

Barucq, C., Ait-Kaci, A., Enrich, J.-J., 2010. Les Services publics d'eau et d'assainissement en France : données économiques, sociales et environnementales. Rapport BIPE, Issy-lesMoulineaux / FP2E, Paris, $4^{\mathrm{e}}$ édition (http://www.fp2e.org [les-publications-de-la-fp2e]).

Burtin, C., Destandau, F., Tsanga-Tabi, M., à paraître. Connaissance et maîtrise des coûts dans le secteur de l'eau potable et de l'assainissement, in Bouleau, G., GuérinSchneider, L. (Eds), Des tuyaux et des hommes : les réseaux d'eau en France, Versailles, Quæ.

Camp, R., 1989. Benchmarking: The Search for Industry Best Practices That Lead To Superior Performance, Milwaukee (WI), Quality Press / White Plains (NY), Quality Resources. Trad. fr. : Le Benchmarking : pour atteindre l'excellence et dépasser vos concurrents, Paris, Éditions d'Organisation, 1992.

Canneva, G., Pezon, C., 2008. Des communes aux communautés, la révolution invisible des services d'eau en France, Flux, 74, 56-67.

Cour des comptes, 1997. La Gestion des services publics locaux d'eau et d'assainissement. Rapport public particulier, Cour des comptes, Paris (http://www.ccomptes.fr/fr/CC/Theme144.html).

Cour des comptes, 2003. La Gestion des services publics d'eau et d'assainissement. Rapport public particulier, Cour des comptes, Paris (http://www.ccomptes.fr/fr/CC/Theme-56. html).

Cousquer, Y., Dumont, J., François, H., Lavoux, T., Prime, J.-L., 2005. Les Indicateurs de performance appliqués aux services publics de l'eau et de l'assainissement : constats et propositions, Paris, La Documentation française.

David, A., 1996. Structure et dynamique des innovations managériales, Cahier de recherche du CGS, 12.

David, A., 2008. La recherche-intervention, cadre général pour la recherche en management?, in David, A., Hatchuel, A., Laufer, R. (Eds), Les Nouvelles Fondations des sciences de gestion, Paris, Vuibert.

Desrosières, A., 2008. La statistique, outil de gouvernement et outil de preuve, in Desrosières, A. (Ed.), Pour une sociologie historique de la quantification : l'argument statistique, 1, Paris, Presses des Mines.

Faucheux, S., Nicolaï, I., 2004. La responsabilité sociétale dans la construction d'indicateurs : l'expérience de l'industrie européenne de l'aluminium, Natures Sciences Sociétés, 12, 1, 30-41.

Giard, V., 2003. La normalisation technique, Revue française de gestion, 29, 147, 49-65.

Guérin-Schneider, L., 2001. Introduire la mesure de performance dans la régulation des services d'eau et d'assainissement en France : instrumentation et organisation. Thèse de gestion, ENGREF, Paris.
Guérin-Schneider, L., Nakhla, M., 2000. Le service public d'eau délégué : du contrôle local des moyens au suivi de la performance, Politiques et management public, 18, 1, 105-123.

Guérin-Schneider, L., Nakhla, M., 2003. Les indicateurs de performance : une évolution clef dans la gestion et la régulation des services d'eau et d'assainissement, Flux, 52-53, 55-68.

Haut Conseil du secteur public, 1999. Quelle régulation pour l'eau et les services urbains? Rapport, Paris, La Documentation française (http://www.ladocumentationfrancaise.fr/rapports-publics/004000299/index.shtml).

Janicot, L., 2007. Les systèmes d'indicateurs de performance environnementale (IPE), entre communication et contrôle, Comptabilité Contrôle Audit, 13, 1, 47-68.

Lakhal, F., 2006. Les mécanismes de gouvernement des entreprises et les publications volontaires des résultats en France, Comptabilité Contrôle Audit, 12, 2, 69-92.

Martinand, C., 2001. La Maîtrise des services publics urbains organisés en réseaux. Rapport du Conseil économique et social, Journaux officiels, Paris (http://www. ladocumentationfrancaise.fr/rapports-publics/064000219/ index.shtml).

Matos, R., Cardoso, A., Ashley, R., Duarde, P., Molinari, A., Schulz, A., 2003. Performance Indicators for Wastewater Services: IWA Manual of Best Practice, London, IWA Publishing.

Moisdon, J.-C., 1984. Recherche et intervention, Revue française de gestion, 47-48, 61-73.

Olivier, D., Moutet, C., 2002. Des normes au service et à la mesure de l'eau, Les Annales des Mines / Réalités industrielles, décembre, 16-21.

Pezon, C., Canneva, G., 2009. Petites communes et opérateurs privés : genèse du modèle français de gestion des services d'eau potable, Espace et sociétés, 139, 21-38.

Pflieger, G., 2002. Domination du consommateur et résistance du citoyen. Les tensions entre les figures de l'usager au sein du système de régulation de l'eau en France, Flux, 48-49, 20-34.

Quairel, F., 2004. Responsable mais pas comptable: analyse de la normalisation des rapports environnementaux et sociaux, Comptabilité Contrôle Audit, 10, 1, 7-36.

Rousseau, S., 2008. Entreprises publiques et développement durable, Revue française de gestion, 5, 185, 47-64.

Sayre, D., 1996. Inside ISO 14000: The Competitive Advantage of Environmental Management, Delray Beach (FL), St. Lucie Press.

Tavernier, Y., 2001. Rapport d'information $n^{\circ} 3081$ déposé par la commission des finances en conclusion des travaux d'une mission d'évaluation et de contrôle par M. Tavernier sur le financement et la gestion de l'eau, Assemblée nationale, Paris (http://www. assemblee-nationale.fr/rap-info/i3081.asp). 